\title{
ON THE FOUNDATIONS OF INVERSION GEOMETRY
}

\author{
BY \\ ALAN J. HOFFMAN
}

Introduction. A 3-dimensional inversion geometry over an ordered field $V$ in which every nonnegative number is a square may be defined as a partially ordered set $\Pi$ of objects called points, circles, spheres, and inversion space with the properties:

(i) if $p$ is any point, then there is an affine geometry whose "points," "lines," "planes," and "3-space" are, respectively, the points of $\Pi$ other than $p$, the circles containing $p$, the spheres containing $p$, and the inversion space;

(ii) the underlying field of this affine geometry is $V$;

(iii) this affine geometry can be made a Euclidean geometry in such a way that the "circles" and "spheres" of the Euclidean geometry are, respectively, the circles of $\Pi$ not containing $p$ and the spheres of $\Pi$ not containing $p$.

The purpose of this paper is to give axioms for $\Pi$ that will be sufficient to establish (i), (ii), and (iii). The only undefined relation is the ordering relation $\leqq$, which means, geometrically, that all our axioms are incidence axioms. There does not seem to be any particular interest in finding alternative statements of (i), so (i) is simply assumed (1.4). Additional assumptions are added (2.11 and 2.12), and the remainder of the paper is devoted to proving that these axioms are sufficient for (ii) and (iii).

The extension of this work to higher dimensions is straightforward, and we have concentrated on the 3-dimensional case for the sake of simplicity. The 2-dimensional case, however, is different in many ways $\left({ }^{1}\right)$, and will be treated in a future paper.

It is rather surprising that the literature contains so few investigations of the foundations of inversion geometry as an autonomous subject $\left({ }^{2}\right)$. Certainly much less is known about the postulates for inversion geometry than for other geometries. The present paper is an effort to remedy this deficiency.

We wish to thank H. S. M. Coxeter, Tong Hing, and E. R. Lorch for their invaluable advice at various stages in the preparation of this manuscript.

1. The first set of postulates. In this section, we postulate that our set

Presented to the Society, December 28, 1948; received by the editors November 5, 1950 and, in revised form, January 20, 1951.

(1) For the most notable difference, see footnote 7.

${ }^{2}$ In [7] (numbers in brackets refer to the bibliography at the end of the paper), Pieri has treated the 3-dimensional case over the real numbers, and [10] contains a discussion by van der Waerden of the 2-dimensional case over a general field. The principal ideas of these papers are given in footnotes 11 and 15. More recently, Petkantschin [6] has discussed the 2-dimensional case over the real numbers, and it is easy to reformulate his postulates so that the only undefined relation is incidence. 
$\Pi$ has the property (i) of the introduction $\left({ }^{3}\right) . \Pi$ is then imbedded in a lattice $\Lambda$, which is, for present purposes, more easily manageable.

1.1 Axıом. $\Pi$ is a set with a binary relation $\leqq$ defined on it.

An element $p \in \Pi$ with the property $x \leqq p$ implies $x=p$ is called a point. We reserve the letters $p, q, r, \cdots, z$ for points.

1.2 Ахгом. If $a \in \Pi$, then there is a point $p$ such that $p \leqq a$.

1.3 Ахтом. If $p \leqq a$ and $a \leqq b$, then $p \leqq b$.

1.4 Axıом. If $p \in \Pi$, then the following subsystem of $I$, under the relation $\leqq$, is a 3-dimensional affine geometry from which the zero element has been deleted: all points other than $p$, and all elements $a$ such that $p \leqq a$, $p \neq a$.

We note some immediate consequences.

(a) Under $\leqq, \Pi$ is a partially ordered set. This follows at once from the properties of points and the fact that an affine geometry is a partially ordered set.

(b) There is an element $I \in \Pi$ such that $a \in \Pi$ implies $a \leqq I$. Let $p$ be a point of $\Pi$. The affine geometry corresponding to $p$ of 1.4 contains a greatest element, which we denote by $I$. We show that $I$ has the required property; that is, $I$ is the greatest element of $\Pi$. If $a \in \Pi$, let $q \leqq a$ (1.2). Let $J$ be the greatest element in the affine geometry corresponding to $q$. By 1.4, we have $p \leqq J \leqq I$ and $q \leqq J \leqq I$. Thus $I=J$ and $a \leqq I$.

(c) We proceed to the imbedding of $\Pi$ in a lattice. For each pair of distinct $p, q$ we adjoin to $\Pi$ the symbol $P_{p, q}$, obtaining a new set $\Pi^{\prime}, \Pi \subset \Pi^{\prime}$. We now extend the relation $\leqq$ to $\Pi^{\prime}$ by the following rules: $p \leqq P_{p, q}, q \leqq P_{p, q}$; if $a \in \Pi$ and $p, q \leqq a$, then $P_{p, q} \leqq a ; P_{p, q} \leqq P_{p, q}$. It is easy to see that $\Pi^{\prime}$ is a partially ordered set under the extended definition of $\leqq$. Further, if we denote by $\Pi^{\prime}(p)$ the subsystem of $\Pi^{\prime}$ consisting of all elements $a \in \Pi^{\prime}$ such that $p \leqq a$, then $\Pi^{\prime}(p)$ is an affine geometry in which $p$ is the zero element, for $\Pi^{\prime}(p)$ is clearly isomorphic to the affine geometry described in 1.4.

$\Pi^{\prime}(p)$ has a unique extension to a projective geometry of the same dimension. We now adjoin to $\Pi^{\prime}$ the "elements at infinity" of the projective extension of $\Pi^{\prime}(p)$, for each $p \in \Pi$, obtaining a set $\Pi^{\prime \prime} \supset \Pi^{\prime}$. We extend the relation $\leqq$ to $\Pi^{\prime \prime}$ by the following rule: if $a, b \in \Pi^{\prime \prime}$ and there is a point $p$ such that $a$ and $b$ are elements in the projective extension of $\Pi^{\prime}(p)$, and if in that projective geometry $a$ is contained (properly or improperly) in $b$, then we say $a \leqq b$. It is clear that $\Pi^{\prime \prime}$ is partially ordered set. Finally, we adjoin to $\Pi^{\prime \prime}$ an element 0 , obtaining a set $\Lambda$, and extend $\leqq$ by the conditions: $0 \leqq 0 ; 0 \leqq a$ for all $a \in \Pi^{\prime \prime} . \Lambda$ is of course a partially ordered set under $\leqq$, and indeed a lattice.

(3) There are many ways to effect this. See [5] and also [1, p. 109, ex. 12]. We shall for the most part follow [1, chap. I] for the general terminology of ordered sets. We assume familiarity with the lattice-theoretic formulation of projective geometry of Birkhoff and Menger. 
Proof. We show that if $a, b \in \Lambda$, then $\Lambda$ contains $a \cup b$ and $a \cap b$. If at least one of $a, b$ is 0 , the result is immediate, so we assume the contrary. Hence, there exist $p$ and $q$ such that $p \leqq a, q \leqq b$. Let us assume first that $p$ and $q$ can be chosen so that $p=q$. Then the existence of $a \cup b$ and $a \cap b$ follows from the fact that a projective geometry is a lattice. The other possibility is that for every choice of $p \leqq a$ and $q \leqq b$, we have $p \neq q$. In this case, it is immediate that $a \cap b=0$, and what remains to be shown is the existence of $a \cup b$. First, $p \cup q$ exists, and $p \cup q=P_{p, q}$; for l.u.b. $(p, q)$ in $\Pi^{\prime}$ is $P_{p, q}$, by its definition, and the successive imbedding of $\Pi^{\prime}$ in $\Pi^{\prime \prime}$ and $\Lambda$ preserves l.u.b.'s. Next, $((p \cup q) \cup a)$ exists, since $p \leqq p \cup q, p \leqq a$. Similarly, $(((p \cup q) \cup a) \cup b)$ exists, and clearly is $a \cup b$.

Henceforth, unless otherwise specified, an "element" is an element of $\Lambda$.

1.5 Some notations and definitions. The expression " $a$ is contained in $b$ " or " $b$ contains $a$ " means $a \leqq b$. " $a$ is properly contained in $b$ " means $a<b$; that is, $a \leqq b, a \neq b . a \cup b$ is called the join of $a$ and $b, a \cap b$ is called the intersection of $a$ and $b$.

$\Lambda$ is clearly a lattice of dimension 5. Using $d($ ) for the dimension function, we have $d(0)=0, d(p)=1$ for all points $p, d(a)=1$ implies $a$ is a point. An element of dimension 2 is called a pair. We reserve the letters $P, Q, R, \cdots, Z$ for pairs. An element of dimension 3 is called a circle. The symbol [ ] will be used to designate a circle in various ways; for example, $[a b]=$ "the circle containing the elements $a, b$ "; or $[a b]=$ "the elements $a$ and $b$ are contained in a circle." The context will clarify the usage. An element of dimension 4 is called a sphere. The symbol \{\} will be used for spheres in the same manner that [ ] is used for circles. $I$, the unique element of dimension 5 , is called the inversion space.

0 and all elements of $\mathrm{II}^{\prime}$ are called ordinary elements of $\Lambda$. All other elements of $\Lambda$ are called singular. Thus, the only singular elements are certain pairs, circles, and spheres; namely, those elements of $\Pi^{\prime \prime}$ that are not elements of $I^{\prime}$. Note that the ordinary pairs are precisely those elements of $\Pi^{\prime}$ that are not elements of $\Pi$.

We shall specify that an element is singular by attaching the unique point it contains as a subscript; for example, $a_{p}$ is a singular element containing $p$. Observe that according to the construction of $\Lambda$ there is one and only one singular sphere containing $p$. Singular spheres will be denoted by capital letters at the beginning of the alphabet. Thus, $A_{p}$ is the unique singular sphere containing $p$. Two ordinary elements of $\Lambda$ are said to be tangent if their intersection is a singular element $\left(^{4}\right)$.

The following statements are obvious:

1.5.1 If $a$ is ordinary, and $d(a)=n \neq 0$, then there exist $p_{1}, \cdots, p_{n}$ such that $p_{1} \cup \cdots \cup p_{n}=a$.

(4) This is precisely the reason for introducing singular elements. Otherwise, we would be bothered in various places to consider tangency as a special case when, in fact, it is not. 
1.5.2 (a) If $a$ is ordinary, $b<a$, then there exists $p \leqq a, p \nless b, p \neq b$.

(b) For any $a \in \Lambda$, if $p \nless a, p \neq a$, then $d(p \cup a)=1+d(a)$.

1.5.3 If $a \bigcap b \neq 0$, then $d(a)+d(b)=d(a \cup b)+d(a \cap b)$.

1.5.4 Every singular element containing $p$ is contained in $A_{p}$. Conversely, if $a \leqq A_{p}, a \neq 0, p$, then $a$ is singular.

1.5.5 If $a$ is an ordinary circle (sphere), $p<a, q \nless a$, then there exists one and only one circle (sphere) containing $p$ and $q$ and tangent to $a$. Such a circle (sphere) is said to be tangent to $a$ at $p$.

1.6.1 Lемма. Every ordinary circle contains an infinite number of points.

Proof. If we use 1.4, any two ordinary circles contain the same number of points (which may be infinite). If this number is finite, say $n+1$, then the number of points on each ordinary sphere is $n^{2}+1$, the number of points of $\Lambda$ is $n^{3}+1$, the number of ordinary spheres containing a given point is $n^{3}+n^{2}$ $+n$. If $D$ is the number of ordinary spheres, then by counting the number of incidences of points with spheres, we have

$$
\left(n^{3}+n^{2}+n\right)\left(n^{3}+1\right)=\left(n^{2}+1\right) D .
$$

From 1.5.1, we know $n>1$; but for $n>1$, this equation is satisfied by no integer $D$.

This lemma will be useful in assuring that we have "enough" points with which to operate. The following theorem, which we shall use a great deal from $\$ 4$ on, is an easy consequence of the fact that every ordinary circle contains at least four points. We omit its proof.

1.6.2 THEOREM. Let $\tau$ be any 1-1 transformation of the set of points of $\Lambda$ onto itself such that $\left[p_{1} p_{2} p_{3} p_{4}\right]$ implies $\left[\tau p_{1} \tau p_{2} \tau p_{3} \tau p_{4}\right]$. Then $\tau$ can be extended uniquely to an automorphism $\tilde{\tau}$ of $\Lambda$.

2. The definition of inversion and the second set of postulates. We now prove a sequence of theorems corresponding to the construction of the ideal point in a four-dimensional incidence geometry $\left(^{5}\right)$. These lay the foundation for the definition of inversion.

2.1 Theorem( $\left.{ }^{6}\right)$. Let $P_{1}, P_{2}, P_{3}$ be distinct pairs, not all contained in a circle, such that $\left[P_{1} P_{2}\right],\left[P_{1} P_{3}\right],\left[P_{2} P_{3}\right]$. Let $p$ be any point not contained in any of these three circles. By 1.5.2(b) and 1.5.3, $\left[p P_{1}\right] \cap\left[p P_{2}\right]$ is a pair, say $P$. Then $\left[\begin{array}{ll}P & P_{3}\end{array}\right]$.

Proof. We first note that $\left\{P_{1} P_{2} P_{3}\right\}$, since by 1.5.3, $d\left(P_{1} \cup P_{2} \cup P_{3}\right)$

(5) Our lattice $\Lambda$ and the semi-lattice of incidence geometry considered by Gorn in [3] are sufficiently similar that the work of [3] is applicable here. (This remark was made in [5]. Its meaning is that an inversion geometry is an example of an incidence geometry.) 2.2, 2.6, and 2.7 are restatements, for present use, of theorems of [3].

${ }^{(6)}$ This is condition $E$ of [3]. The proof of this theorem has been known, although it does not seem to be in the literature. 
$=d\left(\left(P_{1} \cup P_{2}\right) \cup\left(P_{1} \cup P_{3}\right)\right)=d\left(P_{1} \cup P_{2}\right)+d\left(P_{1} \cup P_{3}\right)-d\left(P_{1}\right)=3+3-2=4$. We now consider two cases:

(i) $p \nless\left\{P_{1} P_{2} P_{3}\right\}$. Then $P<\left\{p P_{1} P_{3}\right\} \cap\left\{p P_{2} P_{3}\right\}=\left[p P_{3}\right]$. Hence, $\left[P P_{3}\right]$.

(ii) $\left.p<\left\{P_{1} P_{2} P_{3}\right\}{ }^{7}\right)$. By 1.5.2(a), there exists a point $q \nless\left\{P_{1} P_{2} P_{3}\right\}$. By case (i), $q<Q=\left[\begin{array}{ll}q & P_{1}\end{array}\right] \cap\left[\begin{array}{ll}q & P_{2}\end{array}\right] \cap\left[\begin{array}{ll}q & P_{3}\end{array}\right]$. Now $p \nless Q$, for otherwise, $p=Q$ $\bigcap\left\{P_{1} P_{2} P_{3}\right\}<\left[q P_{1}\right] \cap\left\{P_{1} P_{2} P_{3}\right\}=P_{1}$, contrary to hypothesis. Therefore, $[p Q]$. Further, by 1.5.3, $[p Q] \cap\left\{P_{1} P_{2} P_{3}\right\}$ is a pair, which we denote by $P$. We show that $P$ has the required property. We have $P<\left\{P_{1} P_{2} P_{3}\right\}$ $\cap\left\{p\left[Q P_{i}\right]\right\}$ and $P_{i}<\left\{P_{1} P_{2} P_{3}\right\} \cap\left\{p\left[Q P_{i}\right]\right\}$. Hence, $\left[P P_{i}\right]$ for $i=1,2,3$, which is the result sought.

2.2 Corollary. If $P_{1}$ and $P_{2}$ are distinct pairs such that $\left[P_{1} P_{2}\right], q \nless\left[P_{1} P_{2}\right]$, $r \nless\left[P_{1} P_{2}\right], Q=\left[\begin{array}{ll}q & P_{1}\end{array}\right]\left[q, P_{2}\right], R=\left[\begin{array}{ll}r & P_{1}\end{array}\right] \cap\left[\begin{array}{ll}r & P_{2}\end{array}\right], p<\left[\begin{array}{l}P_{1} P_{2} \\ ]\end{array}\right]$, then $[p Q]$ $\cap[p R] \cap\left[P_{1} P_{2}\right]$ is a pair.

Proof. If $Q=R$, the result is immediate, so let us assume the contrary' We show first that $[Q R]$. If $r<\left[q P_{1}\right]$ or $r<\left[q P_{2}\right]$, this follows at once from the definition of $Q$ and $R$; if $r \varangle\left[q P_{1}\right]$ and $r \varangle\left[q P_{2}\right]$, this follows from 2.1. Applying 2.1 now to $Q, R, P_{1}$ and $p$, we have the corollary, provided [ $Q R P_{1}$ ] is false. If [ $\left.Q R P_{1}\right]$, then apply 2.1 to $Q, R, P_{2}$ and $p$.

2.3 Definition. We say that $P_{1}, P_{2}$ are anallagmatic pairs (of a fundamental involution) if

(i) $\left[P_{1} P_{2}\right]$, and

(ii) $P_{1} \cap P_{2}=0$.

The terminology will be shown to be appropriate in 2.8 .

2.4 Lemma. If $P_{1}, P_{2}$ are anallagmatic pairs, $p \nless\left[P_{1} P_{2}\right], P_{3}=\left[\begin{array}{ll}p & P_{1}\end{array}\right]$ $\cap\left[p P_{2}\right]$, then $P_{1}, P_{3}$ are anallagmatic pairs.

Proof. Condition (i) of 2.3 is satisfied at once. Assume (ii) is not satisfied Then $P_{3} \cap P_{1} \neq 0$ implies that $\left[p P_{1}\right] \cap\left[p P_{2}\right] \cap P_{1}=P_{1} \cap\left[\begin{array}{ll}p & P_{2}\end{array}\right] \neq 0$. Since $p$ $\varangle\left[P_{1} P_{2}\right]$, it follows that $P_{1} \cap\left[p P_{2}\right]$ is a point, say $q$. Therefore, $\left[p P_{2}\right]$ $=\left[q P_{2}\right]=\left[P_{1} P_{2}\right]$, so $p<\left[P_{1} P_{2}\right]$, which violates the hypothesis.

2.5 Theorem. Let $P_{1}, P_{2}$ be anallagmatic pairs. Then there exists a function $F$ with the following properties:

(a) $F$ is mapping of the set of points of $\Lambda$ into the set of pairs of $\Lambda$.

(b) $p<F(p)$.

(c) $P_{1}$ and $P_{2}$ are in the image of $F$.

(d) If $Q \neq R$ are in the image of $F$, then $Q, R$ are anallagmatic pairs.

(7) Although this case involves only objects contained in a sphere, an example due to Hjelmslev (see [2, p. 229], where the example is obviously intended to apply to 2.1 (ii)) shows that its proof requires the use of a point not on the sphere. This striking analogy to the Desargues situation in projective geometry was pointed out in [4], where it was strengthened by showing that Miquel's theorem (see footnote 15) implies 2.1 (ii), as Pappus implies Desargues. 
Proof. If $p<P_{1}$ (or $p<P_{2}$ ), we define $F(p)=P_{1}$ (or $P_{2}$ ). If $p<\left[P_{1} P_{2}\right]$, then we define $F(p)=\left[p P_{1}\right] \cap\left[p P_{2}\right]$. If $p<\left[P_{1} P_{2}\right]$, but $p<P_{1}, p<P_{2}$, then we define $F(p)$ to be the pair discussed in the conclusion of Corollary 2.2.

(a), (b), and (c) are satisfied immediately. (d) is a consequence of Theorem 2.1 and is essentially a summation of all we have done so far in $\$ 2$. We omit a formal proof.

2.5.1 REMARK. $F$ is uniquely determined by any two distinct pairs in its image.

2.6 We now generalize 2.1 in such a way as to lead to a definition and discussion of coaxal circles.

THEOREM. Let $a_{1}, a_{2}, a_{3}$ be distinct circles not all contained in a sphere such that $\left\{a_{1} a_{2}\right\},\left\{a_{1} a_{3}\right\},\left\{a_{2} a_{3}\right\}$. Let $p$ be a point not contained in any of these three spheres. Then there is a unique circle a containing $p$ such that $\left\{a a_{1}\right\},\left\{a a_{2}\right\}$, $\left\{\begin{array}{ll}a & a_{3}\end{array}\right\}$.

Proof. If $a_{1} \cap a_{2} \neq 0$, then $a_{1} \cap a_{2}$ is a pair $P . P<\left\{a_{1} a_{3}\right\} \cap\left\{a_{2} a_{3}\right\}=a_{3}$. The $a$ of the theorem is then $[p P]$.

If $a_{1} \cap a_{2}=0$, then by the above, $a_{1} \cap a_{3}=a_{2} \cap a_{3}=0$. Choose $p_{1}<a_{1}, p_{2}<a_{2}$, $p_{3}<a_{3}$. Further, let $P_{1} \neq Q_{1}$ be chosen so that $p_{1}<P_{1}, p_{1}<Q_{1}, \quad\left[P_{1} Q_{1}\right]=a_{1}$. Let $P_{2}=\left[p_{2} P_{1}\right] \cap a_{2}, Q_{2}=\left[p_{2} Q_{1}\right] \cap a_{2}$. Then the $P$ 's and $Q$ 's determine respectively a function $F_{P}$ and a function $F_{Q}$ in accordance with 2.5 . We note first that $F_{P}\left(p_{3}\right)<a_{3}$. For $F_{P}\left(p_{3}\right)=\left[p_{3} P_{1}\right] \cap\left[p_{3} P_{2}\right]<\left\{p_{3} a_{1}\right\} \cap\left\{p_{3} a_{2}\right\}=\left\{a_{3} a_{1}\right\}$ $\cap\left\{a_{3} a_{2}\right\}=a_{3}$. Similarly, $F_{Q}\left(p_{3}\right)<a_{3}$. Further, if $x$ is any point of $\Lambda$, then $F_{P}(x) \neq F_{Q}(x)$. For if $x \nless a_{1}$, then since $\left[P_{1} F_{P}(x)\right]$, we have $P_{1}=\left[p_{1} F_{P}(x)\right]$ $\cap a_{1}$. Similarly, $Q_{1}=\left[p_{1} F_{Q}(x)\right] \cap a_{1}$. If $F_{P}(x)=F_{Q}(x)$, then $P_{1}=Q_{1}$, which is a contradiction. If $x<a_{1}$, the same argument would establish $P_{2}=Q_{2}$, and this would violate what we have just shown in the case $x=p_{2}$. In particular, $F_{P}(p) \neq F_{Q}(p), F_{P}\left(p_{i}\right) \neq F_{Q}\left(p_{i}\right)(i=1,2,3)$. Let $a=\left[F_{P}(p) F_{Q}(p)\right]$. We shall show that $\left\{\begin{array}{ll}a & \left.a_{i}\right\} \\ \end{array}\right.$ for $i=1,2,3$; that is, $d\left(a \cup a_{i}\right)=4$.

$$
\begin{aligned}
d\left(a \cup a_{i}\right) & =d\left(\left[F_{Q}(p) F_{P}(p)\right] \cup\left[F_{Q}\left(p_{i}\right) F_{P}\left(p_{i}\right)\right]\right) \\
& =d\left(\left[F_{P}(p) F_{P}\left(p_{i}\right)\right] \cup\left[F_{Q}(p) F_{Q}\left(p_{i}\right)\right]\right)=4,
\end{aligned}
$$

by 1.5 .3 and 2.5 (b). The uniqueness of $a$ is obvious.

2.7 Definition. Let $k$ be an ordinary sphere, $a_{1}$ and $a_{2}$ circles contained in $k, p<k, p \nless a_{1} \cap a_{2}, q \nless k$. The circle $a=k \cap\left\{p\left[\left\{q a_{1}\right\} \cap\left\{q a_{2}\right\}\right]\right\}$ is called the circle containing $p$ coaxal with $a_{1}$ and $a_{2}$.

It must be shown, of course, that $a$ does not depend on the choice of $q$. That this is indeed the case can be proven from 2.6 in a manner precisely analogous to the derivation of 2.2 from 2.1 , and we omit the details. It is clear that if $r<a$, then $a$ is also the circle containing $r$ coaxal with $a_{1}$ and $a_{2}$; also, if $a_{1} \cap a_{2} \neq 0$, then the circle containing $p$ coaxal with $a_{1}$ and $a_{2}$ is $p$ $\cup\left(a_{1} \cap a_{2}\right)$.

The set of all circles coaxal with two given circles is called a coaxal set of 
circles. It is clear that any two circles of a coaxal set determine the coaxal set.

2.8 Definitions. Returning to the function $F$ of 2.5 , we see that $F$ induces in a natural way a 1-1 transformation $\tau$ of the set of points of $\Lambda$ onto itself. $\tau$ is defined as follows:

(i) if $F(p)$ is a singular pair, then $\tau p=p$;

(ii) if $F(p)$ is an ordinary pair, $F(p)=p \cup q$, then $\tau p=q$.

As a transformation, $\tau$ is an involution, and we call $\tau$ a fundamental involution. A fundamental involution, then, is a point-point transformation associated with a function $F$ in the prescribed way. Any pair in the image of $F$ is said to be anallagmatic with respect to $\tau$ (or anallagmatic under $\tau$ ), which justifies the terminology of 2.3. It follows from 2.5.1 that a fundamental involution is completely determined by any two distinct anallagmatic pairs.

2.8.1 Definition. If $\tau$ is the fundamental involution associated with a function $F$, then $a$ is said to be anallagmatic with respect to $\tau$ (or anallagmatic under $\tau$ ) if $a$ contains a pair $P$ in the image of $F$.

2.9 Corollaries. We leave the proofs to the reader.

2.9.1 If $a$ and $b$ are anallagmatic with respect to $\tau$, and if $a \cap b \neq 0$, then $a \cap b$ is anallagmatic with respect to $\tau$.

2.9.2 If $k=\{a b\}$ is an ordinary sphere, and if $a$ and $b$ are circles anallagmatic with respect to $\tau$, then every circle coaxal with $a$ and $b$ is also anallagmatic under $\tau$.

2.9.3 If $\tau, \rho$ are distinct fundamental involutions, and $k$ is an ordinary sphere anallagmatic under both $\tau$ and $\rho$, then the circles on $k$ anallagmatic under both $\tau$ and $\rho$ are a coaxal set of circles.

2.9.4 If $a$ is anallagmatic with respect to $\tau$, and $p<a$, then $\tau p<a$.

2.9.5 $\tau$ and $\rho$ will have a common anallagmatic pair if and only if the coaxal circles of 2.9.3 have a common pair (which, of course, will be the common anallagmatic pair).

2.9.6 If $a \neq 0$ is ordinary and not anallagmatic under $\tau, d(a)=n, a=p_{1}$ $\cup \ldots \cup p_{m}$, and $P_{i}$ is the anallagmatic pair containing $p_{i}$, then $d\left(P_{1} \cup \ldots \cup P_{m}\right)=n+1$.

2.10 Definitions. If $\tau$ is a fundamental involution, a point $p$ such that $\tau p=p$ is called a fixed point or double point of $\tau$. A fundamental involution $\tau$ is called a negative inversion if for $a$ and $b$ ordinary circles anallagmatic under $\tau,\{a b\}$, then $a \cap b$ is an ordinary pair. Any other fundamental involution is called a positive inversion, or briefly, an inversion.

The axioms previously given are insufficient, and we add two more. Specifically, we shall require that (positive) inversions possess further properties. Now, inversions have been defined in $\Lambda$, and our axioms should be given as properties of $\Pi$. It is not difficult, however, to define inversion exclusively in terms of $\Pi$, and we leave this to the reader. The axioms of this section are 
then to be considered as axioms of $\Pi$; but their content is precisely the same as if they were given as properties of $\Lambda$, and it is as such that we shall use them.

- $2.11 \operatorname{Axiom}\left({ }^{8}\right)$. If $\tau$ is an inversion, then every anallagmatic ordinary circle contains at least one fixed point of $\tau$.

It follows that a fundamental involution is an inversion if and only if it admits a double point.

2.12 Axıом. If $\tau$ is an inversion, and if $\left[p_{1} p_{2} p_{3} p_{4}\right]$, then $\left[\tau p_{1} \tau p_{2} \tau p_{3} \tau p_{4}\right]$; that is, any inversion satisfies the hypothesis of 1.6.2.

This completes our list of assumptions. It is possible to replace 2.12 by either of the following axioms:

(i) If $\rho$ and $\tau$ are inversions, then $\rho \tau \rho$ is a fundamental involution. (Hence, $\rho \tau \rho$ is an inversion, for if $x$ is any fixed point of $\tau$, then $\rho x$ is a fixed point of $\rho \tau \rho$.

(ii) If $\tau_{1}, \cdots, \tau_{n}$ are inversions, if $\rho=\tau_{n} \cdots \tau_{1}$, and if $x_{1}, x_{2}$, and $x_{3}$ are distinct points such that $\rho x_{i}=x_{i}(i=1,2,3)$, then $x<\left[x_{1} x_{2} x_{3}\right]$ implies $\rho x=x\left({ }^{9}\right)$.

By virtue of 1.6.2, Axiom 2.12 implies that any inversion $\tau$, or any composition of inversions, has a unique extension to an automorphism of $\Lambda$. No confusion will arise if we use the same symbol for the automorphism that we have hitherto used for the point transformation, and henceforth we shall do so. It is convenient to note here a few useful facts about automorphisms of $\Lambda$.

If $\phi$ is an automorphism of $\Lambda$, and

2.12.1 if $a, b, c$ are coaxal circles, then $\phi a, \phi b, \phi c$ are coaxal circles;

2.12.2 if $a_{p}$ is a singular element, then $\phi a_{p}$ is also singular;

2.12.3 if $P, Q, R$ are anallagmatic pairs of a fundamental involution, then $\phi P, \phi Q, \phi R$ are anallagmatic pairs of a fundamental involution;

2.12.4 if $P=p \cup q$, and if $\phi P=P$, then either $\phi p=p$ or $\phi p=q$; if $\phi p=q$, then $\phi q=p$; if $\phi p=p$, then $\phi q=q$.

2.13 THEOREM. Let $k$ be an ordinary sphere anallagmatic under an inversion $\tau$. Then $k$ contains a circle $c$ such that (i) $p<k, \tau p=p$ imply $p<c$; (ii) $p<c$ implies $\tau p=p$; (iii) $c$ is not anallagmatic under $\tau$; (iv) if $a$ is an ordinary anallagmatic circle on $k$, then $a \cap c$ is an ordinary pair. We call $c$ the circle of inversion of $\tau$ on $k$.

Proof. 2.11 implies that there are at least three fixed points of $\tau$ on $k$. We shall show that all fixed points lie on a circle. Assume the contrary, and

${ }^{(8)}$ This is our only "order" axiom, in contrast with the variety of order axioms in [4] and [5]. This assumption compels our field $V$ to have the property described in the introduction. It is quite clear that, conversely, this assumption is satisfied in any inversion geometry over $V$.

(9) (i) is a special case of Miquel's theorem, as stated in 4.17. (ii) is reminiscent of axiom $P$ in projective geometry $[9$, vol. I, p. 95$]$. 
let $x_{1}, x_{2}, x_{3}, x_{4}$ be fixed but not contained in a circle. Then $\left[x_{1} x_{2} x_{3}\right]$ and $\left[\begin{array}{lll}x_{1} & x_{2} & x_{4}\end{array}\right]$ cannot both be anallagmatic, for that would imply $\tau: x_{1} \rightarrow x_{2}$; so at least one of them, say $\left[\begin{array}{lll}x_{1} & x_{2} & x_{3}\end{array}\right]$, is not anallagmatic. By $2.12, p<\left[\begin{array}{lll}x_{1} & x_{2} & x_{3}\end{array}\right]$ implies $\tau p<\left[\begin{array}{lll}x_{1} & x_{2} & x_{3}\end{array}\right]$, so $\tau p=p$, for otherwise $\left[\begin{array}{lll}x_{1} & x_{2} & x_{3}\end{array}\right]$ would be anallag- $\bullet$ matic. Let $r \neq x_{4}, r \varangle\left[\begin{array}{lll}x_{1} & x_{2} & x_{3}\end{array}\right]$ be any point of $k$, and let $c_{1}=\left[\begin{array}{lll}r & x_{4} & x_{1}\end{array}\right], c_{2}$ $=\left[\begin{array}{lll}r & x_{4} & x_{2}\end{array}\right]$. Then $c_{i}$ contains two points of $\left[\begin{array}{lll}x_{1} & x_{2} & x_{3}\end{array}\right]$ or is tangent to $\left[x_{1} x_{2} x_{3}\right]$, so by 2.12 or $2.12 .2, \tau c_{i}=c_{i}$. Hence, 2.12.4 implies $\tau r=r$. Let $[r s t]$ be any circle of $k$ containing $r$ and anallagmatic under $\tau$, and let $\rho$ be the inversion defined by $\rho: s \rightarrow t, r \rightarrow r\left({ }^{10}\right)$. If $u \neq r$ is a fixed point of $\rho$ on $k$, and $a$ is the unique circle (2.9.6) containing $r$ and $u$ anallagmatic under $\rho$, then $a \cap A_{r}$ and $a \cap A_{u}$ are anallagmatic under both $\rho$ and $\tau$. Therefore, $\rho=\tau$ (2.8), which is impossible. Hence, if $c$ is a circle of $k$ containing three fixed points of $\tau$, say $c=\left[x_{1} x_{2} x_{3}\right]$, (i) is proven. (iii) follows from the fact that we can certainly find a point $x \in \Lambda$ such that $x \nless c, \tau x=x$. If $c$ were anallagmatic, $\{x c\}$ would be an anallagmatic sphere whose fixed points were not contained in a circle. (ii) has already been proven, under the temporary (but now verified assumption) that $\left[\begin{array}{lll}x_{1} & x_{2} & x_{3}\end{array}\right]=c$ is not anallagmatic. By 2.11, an ordinary anallagmatic circle on $k$ contains at least one point of $c$, hence it contains two points, or $c$ would be anallagmatic. This proves (iv).

2.14 Lemмa. Assume that we are given a set of coaxal circles on an ordinary sphere $k$ such that if $a$ and $b$ are distinct circles of the set, then $a \cap b=0$. Then there exist exactly two points $p$ and $q$ such that $A_{p} \cap k$ and $A_{q} \cap k$ are circles of the coaxal set.

Proof. There certainly exist no more than two points $p$ and $q$ with the given property. For if $A_{r} \cap k$ is another singular circle of the coaxal set, let $\sigma$ be the inversion under which $A_{p} \cap[p q r]$ and $A_{q} \cap[p q r]$ are anallagmatic pairs. Then by 2.9.2, $A_{r} \cap k$ is anallagmatic under $\sigma$, so $\sigma r=r$. Therefore, $[p \quad q r]$ would be anallagmatic under $\sigma$ and contain three double points. This violates 2.13 (iii).

Now to show that there are at least two points with the specified property. Let $a$ and $b$ be two ordinary circles of the given set of coaxal circles (if such ordinary circles did not exist, then the assertion to be proven would be immediately true). Let $\mu$ be any fundamental involution under which $a$ and $b$ are anallagmatic ( $\mu$ clearly exists). Since $a \cap b=0$, it follows from 2.10 that $\mu$ is an inversion. By $2.13, k$ contains a circle $c$ which is the circle of inversion of $\mu$. $c$ intersects $a$ in two points, say $s_{1}, s_{2} ; c$ intersects $b$ in two points, say $t_{1}, t_{2}$. Let $\nu$ be the fundamental involution determined by $\nu: s_{1} \rightarrow s_{2}, t_{1} \rightarrow t_{2}$. By 2.10, $\nu$ is an inversion, and $c$ is anallagmatic under $\nu$. Hence, $c$ contains two points, $p$ and $q$, which are double points of $\nu$. Therefore, $p$ and $q$ are double

(10) The final sentence of 2.8 shows that $\rho$ is determined by these stipulations, for $s \cup_{t}$ and $[r s t] \cap A_{r}$ are pairs anallagmatic under $\rho$. 
points of both $\mu$ and $\nu$, so $A_{p} \cap k, A_{q} \cap k, a$ and $b$ are anallagmatic under both $\mu$ and $\nu$. By 2.9.3, $p$ and $q$ are the desired points.

2.15 THEOREM. Given any ordinary circle $d$ on a sphere $k$, then there exists a unique inversion under which $k$ is anallagmatic and $d$ is the circle of inversion.

Proof. There cannot be more than one such inversion, for then the singular circles containing the points of $d$ and contained in $k$ would be coaxal, violating 2.14. To show that there is at least one such inversion, let $p, q$, and $r$ be distinct points contained in $d$, and $s$ a point not contained in $k$. Let

$$
S=\left(s \cup\left(A_{p} \cap k\right)\right) \cap\left(s \cup\left(A_{q} \cap k\right)\right) \cap\left(s \cup\left(A_{r} \cap k\right)\right) .
$$

Since $S \cap k=0$, there exists a fundamental involution $\tau$ under which $S$ and $k$ are anallagmatic. By 2.8.1, $\left(s \cup\left(A_{p} \cap k\right)\right)$ is anallagmatic, hence by 2.9.1 $A_{p} \cap k$ is anallagmatic, so $\tau p=p$, and $\tau$ is an inversion. Similarly, $\tau q=q$, $\tau r=r$. Hence, $d$ is the circle of inversion on $k$ of $\tau$.

2.15.1 Definition. We shall find it occasionally convenient, when the anallagmatic ordinary sphere under discussion, say $k$, is well understood, to designate an inversion $\tau$, whose circle of inversion on $k$ is $c$, by $\tau_{c}$.

2.16. THEOREM. Let a be an ordinary circle contained in a sphere $k, r<k$, $r \nless a, s=\tau_{a} r$. Then $A_{r} \cap k, A_{s} \cap k$ and a are coaxal.

Proof. Let $x<a$. By 2.12, 2.12.1, and 2.12.2, if $b$ is the unique circle containing $x$ coaxal with $A_{r} \cap k$ and $A_{s} \cap k$, then $\tau_{a} b=b$. To prove $b=a$, it is suffcient to show that $b$ is not anallagmatic with respect to $\tau_{a}$. Assume the contrary. Then $\left[\begin{array}{rl}r & s\end{array}\right] \cap b$ is singular. Let $\sigma$ be the inversion which has $A_{r}$ $\cap[r s x]$ and $A_{s} \cap[r s x]$ as anallagmatic pairs. Then by 2.9.2, $b$ is anallagmatic under $\sigma$, so by 2.9.1 $[r s x] \cap b$ is also anallagmatic under $\sigma$. Hence, $r, s$, and $x$ are double points of $\sigma$, violating 2.13 .

3. Harmonic sets and orthogonal circles( $\left.{ }^{11}\right)$.

3.1 Definition. If $p, q, r$, and $s$ are distinct, [ $p q r s$ ], and there is an inversion $\tau$ such that $\tau p=p, \tau q=q, \tau r=s$, we say $H(p q, r s)$ (read " $p q, r s$ are a harmonic set"). It is obvious that if $p, q, r$ are given, $s$ is uniquely determined, $s \neq r$ by 2.13 , and we therefore sometimes say: " $s$ is the harmonic conjugate of $r$ with respect to $p$ and $q . "$

\subsubsection{Corollary. If $H(p q, r s)$, then $H(r s, p q)$.}

Proof. Let $k$ be any sphere containing [ $p q r s$ ], and let $a_{r}=A_{r} \cap k, a_{s}=A_{s}$ $\cap k$. By 3.1 and 2.15.1, $k$ contains a circle $a$ such that $\phi_{a} r=s$, and $a \cap[p q r s]$ $=p \cup q$. Let $\tau$ be the inversion given by $\tau: p \rightarrow q, r \rightarrow r$. Then $a_{r}$ and $a$ are anallagmatic under $\tau$, so by 2.16 and 2.9.2, $a_{s}$ is also anallagmatic under $\tau$. Hence $\tau s=s$. By the definition of $\tau$, this implies $H(r s, p q)$.

(11) The axioms of inversion geometry given by Pieri in [7] place principal emphasis on the notion of harmonic sets and arrive at inversions through them. 
3.1.2 Corollary. If $\phi$ is any automorphism of $\Lambda$, and if $H(p q, r s)$, then $H(\phi p \phi q, \phi r \phi s)$.

This follows easily from 2.12 .2 and 2.12.3.

3.2 Definition. If $a$ and $b$ are ordinary circles contained in a sphere $k$, we say $a \perp b$ (read " $a$ is orthogonal to $b$ ") if $b$ is anallagmatic under $\phi_{a}$.

The following statements are immediate:

3.2.1 If $a \perp b$ and $\tau$ is an automorphism of $\Lambda$, then $\tau a \perp \tau b$.

3.2.2 $a \perp b$ implies that $a \cap b$ is an ordinary pair.

3.2.3 If $a$ is an ordinary circle contained in a sphere $k$, and if $p, q$ are distinct points contained in $k$ with $\phi_{a} p \neq q$, then there exists one and only one circle $b$ such that $p, q<b<k$ and $a \perp b$.

3.2.4 If $a$ and $b$ are circles tangent at a point $p$, and if $c$ is a circle such that $p<c<\{a b\}$ and $c \perp a$, then $c \perp b$.

\subsubsection{TheOREM. $a \perp b$ implies $b \perp a$.}

Proof. Let $b \cap a=p \cup q$ (3.2.2). Let $r$ be any other point contained in $a$. Since $a \perp b$, it follows that $\left[r \cup\left(A_{p} \cap b\right)\right]$ is tangent to $\left[r \cup\left(A_{q} \cap b\right)\right]$. Let $\phi_{b} r=s$. It is clear $r \neq s$. By 2.12.2, $\phi_{b} A_{p}=A_{p}$ and $\phi_{b} A_{q}=A_{q}$, so that $\left[s \cup\left(A_{p} \cap b\right)\right]$ is tangent to $\left[s \cup\left(A_{q} \cap b\right)\right]$; that is, $s$ is a fixed point of $\phi_{a}$, or $s<a$. It follows that $a$ is anallagmatic under $\phi_{b}$, since $a$ contains the anallagmatic pair $r \cup s$; hence, $b \perp a$.

3.3 Theorem. If $a$ and $b$ are ordinary circles on $a$ sphere $k$ and $a \perp b$, then $\tau_{a} \tau_{b}=\tau_{b} \tau_{a}$.

Proof. Let $\rho=\tau_{b} \tau_{a} \tau_{b} \tau_{a}$. By 1.6.2, it is sufficient to show that $\rho$ takes every point of $\Lambda$ into itself. Let $a \cap b=p \cup q$. It is clear that $\rho p=p, \rho q=q$. Let $x$ be any other point of $\Lambda$, and let $X$ be the unique pair containing $x$ that is anallagmatic under $\tau_{b}$. We then have by 3.2.5 that $X, A_{p} \cap a, A_{q} \cap a$ are distinct pairs anallagmatic with respect to $\tau_{b}$. Since $A_{p} \cap a$ and $A_{q} \cap a$ are taken into themselves by $\tau_{a}$, it follows from 2.12.3 that $\tau_{a} X, A_{p} \cap a$ and $A_{q} \cap a$ are anallagmatic pairs of a fundamental involution, which clearly is $\tau_{b}$. Hence $\rho X=\tau_{b} \tau_{a} \tau_{b}\left(\tau_{a} X\right)=\tau_{b} \tau_{a}\left(\tau_{a} X\right)=\tau_{b} X=X$. This shows that if $X$ is singular, then $\rho x=x$. If $X$ is ordinary, $X=x \cup y$, say, then $\tau_{a} X=\tau_{a} x \cup \tau_{a} y$. Hence $\rho x=\tau_{b} \tau_{a}\left(\tau_{b} \tau_{a} x\right)=\tau_{b} \tau_{a}\left(\tau_{a} y\right)=\tau_{b} y=x$.

3.4 CoRollary. Under the hypothesis of 3.3, if $a \cap b=p \cup q$, and if $P_{p}$ is $a$ singular pair such that $p<P_{p}<k$, then $\tau_{b} \tau_{a} P_{p}=P_{p}$.

Proof. Assume, temporarily, that if $x<k, x \neq p, q$, then $\tau_{b} \tau_{a} x \neq x$. Let $c$ be any ordinary circle contained in $k$ such that $q \nless c, P_{p}<c$. Let $d=\tau_{b} \tau_{a} c$, so that by 3.3 we have $c=\tau_{b} \tau_{a} d$. If $c=d$ (actually, this cannot occur), then since $p$ is a fixed point of both $\tau_{a}$ and $\tau_{b}$, we have $\tau_{b} \tau_{a} P_{p}=\tau_{b} \tau_{a}\left(A_{p} \cap c\right)=\tau_{b} \tau_{a} A_{p}$ $\bigcap \tau_{b} \tau_{a} c=A_{p} \cap c=P_{p}$. If $c \neq d$, then consider the pair $c \cap d$. It is easy to see that this pair is taken into itself by $\tau_{b} \tau_{a}$. Consequently, if $c \cap d$ is ordinary, say 
$c \cap d=p \cup x$, then by $2.12 .4, \tau_{b} \tau_{a} x=x$, which contradicts our assumption. Therefore, $c \cap d$ is singular, so that $c \cap d=A_{p} \cap c=P_{p}$, and we have $\tau_{b} \tau_{a} P_{p}$ $=P_{p}$.

It remains, then, to prove our assumption. We first note that $A_{p} \cap k$ and $A_{q} \cap k$ are anallagmatic with respect to both $\tau_{a}$ and $\tau_{b}$. Hence, by 2.9.5, $\tau_{a}$ and $\tau_{b}$ do not have a common anallagmatic pair. If $\tau_{a} x=\tau_{b} x$, then $x$ is either a fixed point of both inversions (impossible, since $x \nless a \cap b$ ) or $x$ is contained in an ordinary pair anallagmatic under both inversions, which violates the preceding sentence. Hence, $\tau_{a} x \neq \tau_{b} x$, which is equivalent to our assumption.

3.5 CoRollary. Under the hypothesis of 3.3, if $c$ is any circle such that $a \cap b=p \cup q<c<k$, then $\tau_{b} \tau_{a} c=c$.

Proof. Let $P_{p}=A_{p} \cap c$, so that $c=q \cup P_{p}$. By 3.4, $\tau_{b} \tau_{a} c=\tau_{b} \tau_{a}\left(q \cup P_{p}\right)$ $=\tau_{b} \tau_{a} q \cup_{\tau_{b}} \tau_{a} P_{p}=q \cup P_{p}=c$.

3.6 CoRollary. Under the hypothesis of 3.3 , if $x$ is a point contained in $k$ but $x \neq p, q$, then $H\left(p q, x \tau_{b} \tau_{a} x\right)$.

Proof. That $x$ is distinct from $\tau_{b} \tau_{a} x$ was shown in 3.4 and [ $p q x \tau_{b} \tau_{a} x$ ] follows from 3.5. By 2.9.2, if $c$ is the circle containing $x$ coaxal with $A_{p} \cap k$ and $A_{q} \cap k$, then $\tau_{b} \tau_{a} x<c$. If $\sigma$ is the inversion under which $A_{p} \cap[p q x]$ and $A_{g} \cap[p q x]$ are anallagmatic pairs, then by 2.13 and 2.12 .4 , we have $\sigma: p \rightarrow p, q \rightarrow q, x \rightarrow \tau_{b} \tau_{a} x$. This is equivalent to the statement to be proven.

3.7 CoRollary. If $a$ and $b$ are ordinary circles tangent at a point $p$, and if $P_{p}$ is a singular pair such that $p<P_{p}<\{a b\}$, then $\tau_{b} \tau_{a} P_{p}=P_{p}$.

Proof. Let $c<\{a b\}$ be a circle orthogonal to $a$ and containing $p$. By 3.2.4, $c \perp b$. Note that $\tau_{b} \tau_{a}=\tau_{b} \tau_{a}\left(\tau_{c} \tau_{c}\right)=\left(\tau_{b} \tau_{c}\right)\left(\tau_{a} \tau_{c}\right)$, by 3.3. An application of 3.4 completes the proof.

3.8 COROLLARY. On an ordinary sphere $k$, there do not exist more than three mutually orthogonal circles.

3.9 THEOREM. If $k$ is a sphere anallagmatic under a negative inversion $\tau$, then the restriction to $k$ of $\tau$ is the composition of (positive) inversions.

The theorem is actually true throughout $\Lambda$, not merely on $k$. But the stronger result, which would require some preparation, is not needed for what follows.

Proof. By $2.11, k$ is an ordinary sphere, and if $a$ is any circle contained in $k$ anallagmatic with respect to $\tau$, then $a$ is also ordinary. Consider $\phi_{a}$. The circles on $k$ anallagmatic under both $\tau$ and $\phi_{a}$ form a coaxal set of circles which by 2.10 have an ordinary pair, say $p \cup q$, in common. By 2.9.5, $p \cup q$ is anallagmatic with respect to both $\tau$ and $\phi_{a}$.

In order to prove the theorem, it will suffice to prove that for every $x<k$, 
$x \neq p, q$, the point $\tau x$ is the harmonic conjugate of $\phi_{a} x$ with respect to $p$ and $q$. For assume that this has been shown. Let $c$ and $d$ be two orthogonal circles on $k$, each containing $p$ and $q$. Then by 3.6, we have $\tau x=\phi_{d} \phi_{c} \phi_{a} x$. Further, $p$ and $q$ will be double points of both $\phi_{d}$ and $\phi_{c}$, so that $\tau=\phi_{d} \phi_{c} \phi_{a}$ for every point of $k$.

Now to prove our statement, let $b$ be the circle containing $p$ and $q$ orthogonal to $[p q x]$ (3.2.3). Since $p \cup q<b$, and $p \cup q$ is anallagmatic under $\phi_{a}$, it follows that $a \perp b$. Therefore, $a \cap b$ is an ordinary pair, say $s \cup t$; and since $a$ and $b$ are anallagmatic under $\tau$, so is $s \cup t$. The harmonic conjugate of $\phi_{a} x$ with respect to $p$ and $q$ is clearly $\phi_{b}\left(\phi_{a} x\right)$. Because $a \perp b$, we have (3.5) $\left[x \phi_{b} \phi_{a} x s t\right]$. Because $[p q x]$ is anallagmatic under both $\phi_{a}$ and $\phi_{b}$, we have $\left[\begin{array}{lll}x \phi_{b} \phi_{a} x & p & q\end{array}\right]$. Therefore, since $p \cup q$ and $s \cup t$ are anallagmatic with respect to $\tau$, it follows that $x \cup \phi_{b} \phi_{a} x$ is anallagmatic with respect to $\tau$. This, however, is equivalent to the statement to be proved.

An obvious and important consequence is that $\tau$ has a unique extension to an automorphism of the sublattice of $\Lambda$ consisting of all elements contained in $k$, which can be further extended to an automorphism of $\Lambda$. No confusion will arise when we use the same symbol for the automorphism as for the point transformation.

3.10 Lemma. Let $k$ be an ordinary sphere, a an ordinary circle contained in $k$. Let $\phi$ be an automorphism of $\Lambda$ such that $\phi k=k$, and $\phi x=x$ for all $x<a$. Then, restricted to $k, \phi$ is either the identity mapping or $\phi_{a}$.

Proof. It may be that $k$ contains a point $p$ such that $p \nless a$ and $\phi p=p$. Let $q$ be any other point of $k$ not contained in $a$. Let $b$ and $c$ be distinct circles, each containing $p, q$ and a point of $a$. It is clear that $\phi b=b, \phi c=c$, so that $\phi(b \cap c)=b \cap c$. By 2.12.4, we have $\phi q=q$, so that in this case $\phi$, restricted to $k$, is the identity map.

On the other hand, it may be that if $p<k, p \nless a$, then $\phi p \neq p$. Let $b_{1}, b_{2}$ be circles such that $p<b_{i} \perp a(i=1,2)$. Let $b_{i} \cap a=x_{i} \cup y_{i}$. By 3.2.1, $\phi b_{i}$ is a circle contained in $k$ orthogonal to $a$, and containing $x_{i}$ and $y_{i}$, so that by 3.2.3, $\phi b_{i}=b_{i}$. Since $b_{1} \cap b_{2}=p \cup \phi_{a} p$, an application of 2.12 .4 shows that in this case $\phi$, restricted to $k$, is $\phi_{a}$.

3.11 ThEOREM. If a pair $P$ is anallagmatic under three (not necessarily distinct) fundamental involutions $\tau_{1}, \tau_{2}, \tau_{3}$, then $\tau_{3} \tau_{2} \tau_{1}$ is a fundamental involution.

Proof. Case (i). $P$ is an ordinary pair, say $P=p \cup q$. Let $r$ be any point of $\Lambda$ other than $p$ or $q$. It is clear that $\left[p q r \tau_{3} \tau_{2} \tau_{1} r\right]$. Let $\rho$ be the fundamental involution defined by $\rho: p \rightarrow q, r \rightarrow \tau_{3} \tau_{2} \tau_{1} r$, and let $\phi=\rho \tau_{3} \tau_{2} \tau_{1}$. It is clearly sufficient to show that if $s$ is any point of $\Lambda$, then $\phi s=s$. Let $k$ be any sphere containing $p, q, r, s$. Let $b$ be the circle on $k$ containing $p$ and $r$ and orthogonal to $[p q r]$ (3.2.3). Since $\phi p=p, \phi r=r$ and $\phi[p q r]=[p q r]$, it follows 
from $2.12,3.9$, and 3.2.1 that $\phi b=b$. Let $t$ be any point contained in $b$ other than $p$. Since $\phi[t p q]=[t p q]$ and $b \cap[t p q]=p \cup t$, it follows from 2.12.4 that $\phi t=t$. Further, since $\phi q=q$ and $q \nless b$, it follows from 3.10 that $\phi s=s$.

Case (ii). $P$ is a singular pair, say $P=P_{p}$. Let $r$ be any point of $\Lambda$ other than $p$, and let $\rho$ be the inversion with respect to which $P_{p}$ is anallagmatic, and which takes $r$ into $\tau_{3} \tau_{2} \tau_{1} r$. If we let $\phi$ designate the automorphism $\rho \tau_{3} \tau_{2} \tau_{1}$, it is sufficient as in Case (i) to show that if $s$ is any point of $\Lambda$, then $\phi s=s$. Let $k$ be any sphere containing $P_{p}, r$, and $s$. As in Case (i), if $b$ is the circle on $k$ that contains $r$, and $p$ is orthogonal to $\left[r P_{p}\right]$, then $\phi$ takes every point of $b$ into itself. We need only consider, then, the case that $s \nless b$. Note that the circles of inversion of $\tau_{1}, \tau_{2}, \tau_{3}$, and $\rho$ have a singular pair containing $p$ in common, since each of these circles contains $p$ and is orthogonal to $\left[r P_{p}\right]$. Hence by 3.7 , if $Q_{p}$ is any singular pair such that $p<Q_{p}<k$, then $\phi Q_{p}=Q_{p}$.

Let $t$ and $u$ be distinct points contained in $b$ other than $p$. Then $[t s p]$ $=t \cup Q_{p}$, where $Q_{p}=A_{p} \cap[t s p]$. By the preceding paragraph, $\phi[t s p]$ $=Q\left(t \cup Q_{p}\right)=\phi t \cup \phi Q_{p}=t \cup Q_{p}=[t s p]$. Similarly, $\phi\left[\begin{array}{lll}u & s & p\end{array}\right]=\left[\begin{array}{lll}u & s\end{array}\right]$. An application of 2.12.4 proves that $\phi s=s$.

4. Coordinates on a circle. We begin the process of introducing coordinates, by establishing a field of points contained in any ordinary circle. This is closely analogous to the well known field of points of a conic $\left({ }^{12}\right)$, with 3.11 playing the role of Pascal's theorem. Then we show that this field is an ordered field in which every positive number is a square.

Let $c$ be any ordinary circle, and let three distinct points contained in $c$ be identified by the labels $0,1, \infty$. (The context will always enable us to distinguish the point 0 from the 0 element of $\Lambda$.)

4.1 Definition. Let $x, y<c ; x, y \neq 0, \infty$. Let $\phi$ be the fundamental involution given by $\phi: 0 \rightarrow \infty, x \rightarrow y$, and let $z=\phi 1$. We say that $z=x y$ ( $z$ is $x$ multiplied by $y$ ). Note that we do not require that $x$ and $y$ be distinct.

4.2 Definition. Let $x, y<c ; y \neq \infty$. Let $\phi$ be the inversion under which $c$ is anallagmatic and $\phi: x \rightarrow y, \infty \rightarrow \infty$. Let $z=\phi 0$. We say that $z=x+y$ ( $z$ is $x$ added to $y$ ). Note that $x$ and $y$ need not be distinct.

4.3 TheOREM. The points of $c$ other than 0 and $\infty$ constitute an abelian group under multiplication. The points of $c$ other than $\infty$ constitute an abelian group under addition.

Proof. We shall prove the statement about multiplication; the proof for addition is essentially the same. It is clear that all we need show is associativity, since all the other postulates for an abelian group are clearly satisfied. Let $x, y, z$ be arbitrary (not necessarily distinct) points contained in $c$ other than 0 and $\infty$. Let

(12) See [9, vol. I, p. 231]. 


$$
\begin{aligned}
& \phi_{1}: x \rightarrow y, \quad 0 \rightarrow \infty, 1 \rightarrow x y, \\
& \phi_{2}: y \rightarrow z, \quad 0 \rightarrow \infty, 1 \rightarrow y z, \\
& \phi_{3}: z \rightarrow x y, 0 \rightarrow \infty .
\end{aligned}
$$

We shall show that $\phi_{3} y z=x$, which is equivalent to $(x y) z=x(y z)$.

By $3.11, \phi_{3} \phi_{2} \phi_{1}$ is an involution. Since $\phi_{3} \phi_{2} \phi_{1} x=x y$, it follows that $x=\phi_{3} \phi_{2} \phi_{1} x y=\phi_{3} \phi_{2} 1=\phi_{3} y z$.

4.4 Definition. If $x \neq \infty$, we say $0 x=x 0=0 ; \infty+x=x+\infty=\infty$. If $x \neq 0$, we say $\infty x=x \infty=\infty$.

4.5 ThEOREM. If $x \neq 0, \infty$, then there exist two fundamental involutions $\phi_{1}, \phi_{2}$ such that for all $p<c$, we have $p x=\phi_{2} \phi_{1} x$. In other words, multiplication by a point other than 0 or $\infty$ is the restriction to $c$ of a lattice automorphism. Similarly, addition by a point other than $\infty$ is the restriction to $c$ of a lattice automorphism.

Proof. For the same reason as in 4.3, we confine our attention to the statement about multiplication. Let $\phi_{1}: 0 \rightarrow \infty, 1 \rightarrow 1$, and let $\phi_{2}: 0 \rightarrow \infty, 1 \rightarrow x$. Assume first that $p \neq 0, \infty$. Then $\phi_{2} \phi_{1} p=\phi_{2} p^{-1}=y$, where $p^{-1} y=x$; that is, $y=p x$, which was to be proven. If $p=0$, we have $\phi_{2} \phi_{1} 0=0=0 x$, by 4.4. The case $p=\infty$ is handled in the same way.

4.6 THEOREM. The points of $c$ other than $\infty$ constitute a field under the given definitions of addition and multiplication.

Proof. In view of 4.3 , all we need show is: if $x, y, z<c$, and $x, y, z \neq \infty$, then $x(y+z)=x y+x z$. If at least one of $x, y, z$ is 0 , the result is immediate, so we assume the contrary. Further, assume temporarily that $y \neq z$ and that $0 \neq y+z$. By 4.2 , the pairs $A_{\infty} \cap c, y \cup z$ and $0 \cup(y+z)$ are anallagmatic pairs of an inversion. By 4.5 and 2.12.3, it follows that $A_{\infty} \cap c, x y \cup x z$, and $0 \cup x(y+z)$ are anallagmatic pairs of an inversion; that is, $x(y+z)=x y+x z$.

If $y=z$, then replace the pair $y \cup z$ in the preceding discussion by $A_{y} \cap c$. If $0=y+z$, then replace the pair $0 \cup(y+z)$ by $A_{0} \cap c$. In each case, the remainder of the proof is essentially the same.

4.7 Definition( $\left.{ }^{13}\right)$. Let $p, q, r, s$ be distinct and [ $\left.p q r s\right]$. Let $\phi$ be the fundamental involution given by $\phi: p \rightarrow q, r \rightarrow s$. If $\phi$ is a negative inversion, we say $p q \mid r s$ ( $p$ and $q$ separate $r$ and $s$ ). If $\phi$ is an inversion, we say $p q \nmid r s$ ( $p$ and $q$ do not separate $r$ and $s$ ).

Observe that separation (or nonseparation) of pairs of points is preserved by any automorphism of $\Lambda$. Further, observe that the field of points on a circles furnishes an easy criterion for separation. Let us set $p=0, q=\infty, r=1$. Then $p q \mid r s$ if and only if $s \neq p, q, r$ and $s$ is not a square in this field. $p q \nmid r s$ if and only if $s \neq p, q, r$ and $s$ is a square in this field.

(13) This definition comes from [7]. 
4.8 Theorem. If $p q \mid r s$, then $p r \nmid q s$.

Proof. Let $a=[p q r s]$, and $k$ be any sphere containing $a$. Let $b$ and $c$ be circles contained in $k$ such that $p \cup q<b \perp a, r \cup s<c \perp a$. By hypothesis, $b\urcorner c$ is an ordinary pair, say $b \cap c=t \cup u$.

Now $p r\{q s$ if and only if the fundamental involution determined by $\tau: p \rightarrow r, q \rightarrow s$ has a double point. But $\tau$ clearly interchanges $b$ and $c$ by 3.2.1, so that $\tau: t \cup u=t \cup u$. Further, $t \cup u$ is not anallagmatic under $\tau$, for that would imply that $b$ is anallagmatic under $\tau$, which is impossible. Hence $\tau t \neq u$, which by 2.12 .4 implies $\tau t=t$. Hence $p r \nmid q s$.

4.9 Theorem. If $p q \nmid x y, q r \nmid x y$, and $p \neq r$, then $p r \nmid x y$.

Proof. It is clear that $[p q r x y]$. We designate this circle by $c$. Set $x=0, y=\infty, q=1$, which determines a field of points contained in $c$. We work in this field. $p q \nmid x y$ implies that there exists $t<c$ such that $t^{2}=p$. $q r \nmid x y$ implies that there exists $u<c$ such that $u^{2}=r$. Then $(t u)^{2}=p r$. Let $\phi$ be the fundamental involution determined by $\phi: p \rightarrow r, 0 \rightarrow \infty$. Then $\phi: 1 \rightarrow p r, t u \rightarrow t u$. Hence $p r \nmid 0 \infty$; that is, $p r \nmid x y$.

4.10 Corollary. If $p q \mid x y$ and $q r \nmid x y$, then $p r \mid x y$.

4.11 Corollary. If $p q|x y, q r| x y$, and $p \neq r$, then $p r \nmid x y$.

Proof. $p q \mid x y$, so by $4.8, p x \nmid q y$ and $p y \nmid q x$. Secondly, $q r \mid x y$, so by 4.8, $r x \nmid q y$ and $r y \nmid q x$.

By 4.9, we have $p r \nmid q y$ and $p r \nmid q x$. Applying 4.9 again, we have $p r \nmid x y$.

4.12 Theorem. If $H(p q, r s)$, then $p q \mid r s$.

Proof. Let $a=[p q r s], k$ be any sphere containing $a$. Since $H(p q, r s)$, $k$ contains a circle $b$ such that $p \cup q<b$ and $\phi_{b} r=s$. Next, consider the fundamental involution $\tau: p \rightarrow q, r \rightarrow s$. $b$ is anallagmatic under $\tau$, so if $\tau$ were an inversion, then $b$ would contain a double point of $\tau$, say $t$. But $r \cup s$ is also anallagmatic under $\tau$, so $[r s t]$ would be tangent to $b$. Since $[r s t]$ is also anallagmatic under $\phi_{b}$, we would have a violation of 2.13 . Hence $\tau$ is a negative inversion, which proves the theorem.

4.13 TheOREM. Let $a$ be any ordinary circle, $0,1, \infty$ three distinct points contained in $a$, and let $F$ be the field of points determined by $0,1, \infty$. Then $F$ is an ordered field in which $x \geqq 0$ if and only if $x$ is a square.

Proof. It is easy to see that it is sufficient to show that $F$ satisfies:

(i) -1 is not a square, and

(ii) if $x$ is not a square and $y$ is not a square, then $x+y$ is not a square $\left({ }^{14}\right)$.

${ }^{(14)}$ This was pointed out in [8], which seems to be the first place where fields of this type were explicitly discussed. 
(i) follows from 4.12, since $H(0 \infty, 1-1)$. To prove (ii), consider first the case $x=y$, so that $x+y=2 x$. From the definition of addition, we have $H(x \infty, 02 x)$, so by $4.12 x \infty \mid 02 x$, which implies (4.8) $x 2 x \nmid 0 \infty$. By hypothesis, $x 1 \mid 0 \infty$, so that by $4.10,12 x \mid 0 \infty$, which was to be proven.

The other case is $x \neq y$. By hypothesis, $x 1|0 \infty, y 1| 0 \infty$, so by 4.11 we have Since $H(\infty(x+y) / 2, x y)$, we have By 4.10 and 4.8 , (a) and (b) imply By 4.8 alone, (b) implies

(a) $x y \nmid 0 \infty$.

(b) $x y \mid(x+y) / 2 \infty$. By 4.9 , (c) and (d) yield Combined with the hypothesis, (e) gives (c) $x(x+y) / 2 \nmid 0 y$. (d) $x(x+y) / 2 \nmid y \infty$. (e) $x(x+y) / 2 \nmid 0 \infty$. Hence, $(x+y) / 2$ is not a square, so, by the previous case, $x+y$ is not a square.

4.14 We now head toward a proof of Miquel's theorem (4.17). We assume that the reader is familiar with the linear fractional transformation from projective geometry, and can prove, using the same ideas as in projective geometry, that if $a=\left[\begin{array}{lll}0 & 1 & \infty\end{array}\right]$ is a circle on a sphere $k$, if $p, q, r, s$ are points of $a$ other than $\infty$, and $p s-q r \neq 0$, then $x^{\prime}=(p x+q) /(r x+s)$ is the restriction to $a$ of an automorphism $\phi$ such that $\phi k=k, \phi a=a$.

4.15 Lemma. Let $k$ be an ordinary sphere, $a$ an ordinary circle contained in $k$. Let $\phi$ be an automorphism of $\Lambda$ such that $\phi k=k$, and let $b=\phi a$. Then, restricted to $k, \tau_{a}=\phi^{-1} \tau_{b} \phi$.

The proof follows readily from 3.10 , and is left to the reader.

4.16 Lemma. Let $\tau$ be an inversion under which $a=\left[\begin{array}{lll}0 & 1 & \infty\end{array}\right]$ is anallagmatic, and let $r$ and $s$ be the double points of $\tau$ on a. Assume $r, s \neq \infty$. Then $\tau$, restricted to $a$, is given by: $x^{\prime}=((r+s) x-2 r s) /(2 x-(r+s))$.

Proof. Let $k$ be any sphere containing $a$. Let $b$ be the circle of inversion of $\tau$. By 4.14, there is an automorphism $\phi$ such that $\phi k=k, \phi a=a$, and $\phi$ is given on $a$ by: $x^{\prime}=(x-r) /(x-s)$. If $c=\phi b$, then $c$ is the circle containing 0 and $\infty$ orthogonal to $a$, so that $\tau_{c}$ is given on $a$ by: $x^{\prime}=-x$. The rest of the proof consists of an application of 4.15 .

4.17 Miquel's TheOREM( $\left.{ }^{15}\right)$. If $\phi_{1}, \phi_{2}, \phi_{3}$ are fundamental involutions such that there exist distinct circles $a$ and $b$ each anallagmatic under $\phi_{i}(i=1,2,3)$, then $\phi_{3} \phi_{2} \phi_{1}$ is a fundamental involution.

Proof. If the three fundamental involutions are the same, the theorem is immediate, so we assume the contrary. This implies $\{a b\}$. Hence, either

(15) In [10], Miquel's Theorem is taken as an axiom, and is the only assumption other than the planar analogue of (i) of our introduction. It is given in the following form: "let $p, q, r, s, t, u$, $v, w$ be distinct points such that [pqrs], [tuvw], [ptuq], [qurv], [rvws]]; then [swtp]." It is then shown that this implies that the affine plane "over" a point satisfies Pappus's Theorem, and that the circles are a family of conics in that affine plane. 
$a \cap b$ is a pair (which case was treated in 3.11), or $a \cap b=0$. We now consider the latter case. Let $k=\{a b\}$. The $\phi_{i}$ are clearly inversions, and by 2.9.3, 2.9.2, 2.9.5, and 2.14, $k$ contains two distinct points $p$ and $q$ such that $k \cap A_{p}$ and $k \cap A_{q}$ are anallagmatic under each $\phi_{i}$. Let $c$ be any ordinary circle contained in $k$ anallagmatic under each $\phi_{i}$. Then $p, q \nless c$, and $c$ contains two double points of each of the given inversions.

In what follows, we assume $\phi_{1}, \phi_{2}, \phi_{3}$ are distinct; the cases in which they are not are easily handled. Let $0, \infty$ be the double points of $\phi_{1}$ on $c ; 1, s$ be the double points of $\phi_{2}$ on $c$. Note that $s$ must be negative, since the fundamental involution $\tau$ given by $\tau: 0 \rightarrow \infty, 1 \rightarrow s$ has $p \cup q$ as an anallagmatic pair, and $H(0 \infty, p q)$. If $r$ is one of the double points of $\phi_{3}$, then the other double point is clearly $s / r$. Let $\phi=\phi_{3} \phi_{2} \phi_{1}$. Applying 4.16, and examining the linear fractional transformation which describes the effect of $\phi$ on $c$, we see that there is an inversion $\rho$ such that $\rho \phi$ is the identity on $c$ and the circle of inversion of $\rho$ contains $p$. Since $p<c$, it follows from 3.10 that $\rho \phi$ is the identity on $k$.

Let $t$ be any point of $\Lambda$ not contained in $k$. Then, letting $d$ be the circle $\{t a\} \cap\{t b\}, d$ is also anallagmatic with respect to the three given inversions. We have $\{c d\}$ because of the definition of coaxal circles. The previous reasoning now shows that $\rho \phi$ is the identity on $\{c d\}$ (note that the definition of $\rho$ does not depend on the sphere containing $c$ ). This completes the proof.

5 . Coordinates on a sphere and throughout $\Lambda$. In this section, we complete the proof that our axioms are sufficient to establish (ii) and (iii) of the introduction.

5.1 We first define the field $V$. We have previously defined the field of points contained in an ordinary circle, which consisted of a set of points and the defined laws of composition, addition and multiplication. We shall define $V$ so that given any of the fields of points previously described, we have a natural isomorphism of $V$ onto this field.

Let $S$ be a set of fields $F$ of points contained in circles and isomorphisms $f$ of these fields, $S=\{F, f\}$, satisfying:

(i) if $F \in S$ and $G \in S$, then there is an isomorphism $f \in S$ such that $f: F \approx G$;

(ii) if $f \in S$ and $F \in S$, and if $f: F \approx F$, then $f$ is the identity map of $F$ onto itself;

(iii) if $f \in S$ and $g \in S$, and if the range of $f$ is the domain of $g$, then $g f \in S$;

(iv) (a geometric condition) we first define: if $F$ is the field of $\left[\begin{array}{lll}0 & 1 & \infty\end{array}\right]$ and $G$ is the field of $\left[\begin{array}{lll}0 & 1 & \infty\end{array}\right]$ (same 0 and $\infty$ ), and if there is an inversion $\phi$ such that $\phi: 0 \rightarrow 0,1 \rightarrow 1^{\prime}, \infty \rightarrow \infty$, then $F$ is said to be $\phi$-related to $G$. We now require of our set $S$ that if $F \in S$ and $G \in S$, and if $F$ is $\phi$-related to $G$, then the restriction of $\phi$ to $F$ is an isomorphism of the set $S\left({ }^{16}\right)$.

There exists at least one set $S$, for example, a single $F$ and the identity

(16) The usefulness of this condition will be seen in 5.4 (iii). 
map. It follows by Zorn's lemma that there exists a maximal set $S$ (which we denote by $\bar{S}$ ), and it will be shown in 5.10 that every $F \in \bar{S}$. We proceed with the construction of $V$.

Consider the $F$ 's in $\bar{S}$ as disjunct sets of elements; that is, points with labels attached to indicate the field. Let $U$ be the set-theoretic union of all elements of all $F$ in $S$. If $x, y \in U$, we define $x \sim y$ if there is an isomorphism $f \in \bar{S}$ such that $f x=y . \sim$ is an equivalence relation in $U$, and each equivalence class $\{x\}$ and each $F$ have exactly one element in common. The equivalence classes form a field $V$ in an obvious way, and if we define $\pi_{F}\{x\}=\{x\} \cap F$, then $\pi_{F}: V \approx F\left({ }^{17}\right)$.

5.2 Let $\infty$ be any point of $\Lambda$, and let $k$ be any ordinary sphere containing $\infty$. In the affine geometry "over" $\infty$ of 1.4 , whose terminology we now adopt, $k$ is a plane. An ordinary circle containing $\infty$ is called a line, and tangent lines are said to be parallel. We proceed to "coordinatize" $k$ in the usual manner. Let 0,1 be two other points contained in the plane $k$, and call the

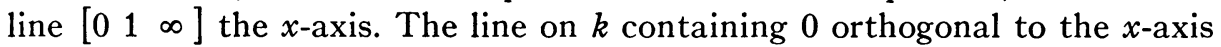
is called the $y$-axis. Of the two inversions that map the $x$-axis onto the $y$-axis, we select one, say $\phi$, and consider the field $G$ of $[0 \phi 1 \infty]$ on the $y$-axis. If we let $F$ be the field of $\left[\begin{array}{lll}0 & 1 & \infty\end{array}\right]$, then by 5.1 we have isomorphisms $\pi_{F}: V \approx F$, $\pi_{G}: V \approx G$. The 1-1 correspondence between ordered pairs of elements of $V$ and points of the plane $k$ (other than $\infty$ ) is given as follows: if $a, b \in V$, then $(a, b)$ corresponds to $p$, where $p \cup_{\infty}$ is the intersection of the line containing $\pi_{F} a$ orthogonal to the $x$-axis and the line through $\pi_{G} b$ orthogonal to the $y$-axis.

5.3 We prove that the points $(a, b)$ constitute a field under the definitions to be given (5.3.2 and 5.3.3) of addition and multiplication. For this work, the following trivial lemma is helpful.

5.3.1 Lemma. Let $K$ be nonempty set, $\{f\}$ a set of 1-1 mappings of $K$ onto itself, and let $K$ and $\{f\}$ satisfy the following conditions:

(i) there is a fixed 1-1 correspondence between the sets $K$ and $\{f\}$. We designate the mapping $f \in\{f\}$ which is associated, in this fixed correspondence, with the element $x \in K$ by $f_{x}$;

(ii) $f_{y} f_{x}=f_{x} f_{y}$;

(iii) $K$ contains an element $e$ such that $f_{x} e=x$, for all $x \in K$.

Then, if we define $x \circ y=f_{x} y, K$ is an abelian group under $\circ$.

Proof. That $e$ is a right unit and that every $x$ has a right inverse is obvious. The operation $\circ$ is commutative, since $x \circ y=f_{x} y=f_{x} f_{y} e=f_{y} f_{x} e=f_{y} x=y$ $\circ x$. The proof is completed by showing that $\mathrm{O}$ is associative:

${ }^{(17)}$ Our definition of the underlying field contains a certain element of arbitrariness, namely in the choice of the maximal $\bar{S}$. An alternative, and possibly superior, approach is to let the field $F$ on the $x$-axis (see 5.2) play a forward role, prove that the axioms are sufficient for inversion geometry, and then define $\bar{S}$ to consist of all fields $F$ and all isomorphisms $f$ arising from composition of inversions. This set will satisfy (i), (ii), (iii), and (iv) by virtue of 2.12 (ii). 
$x \circ(y \circ z)=x \circ(z \circ y)=f_{x} f_{z} y=f_{z} f_{x} y=z \circ(x \circ y)=(x \circ y) \circ z$.

5.3.2 Addition. Let $p$ be any point contained in $k$ other than $\infty$. Express $p$ in terms of its coordinates, say, $p=(a, b)$. Let

$\phi_{1}$ be the inversion "on" $y=0$ (that is, the $x$-axis is the circle of inversion of $\left.\phi_{1}\right)$;

$\phi_{2}$ be the inversion on $y=b / 2$;

$\phi_{3}$ be the inversion on $x=0$;

$\phi_{4}$ be the inversion on $x=a / 2$.

Let $\alpha_{p}=\phi_{4} \phi_{3} \phi_{2} \phi_{1}$. Then the points of $k$ other than $\infty$ and the transformations $\alpha$ (regarded as transformations of the set of points of $k$ other than $\infty$ ) fulfill the conditions of 5.3.1.

Proof. $(0,0)$, or briefly, 0 , clearly plays the role of $e$ in 5.3.1. All we need prove, then, is that if $p, q$ are points of $k$ other than $\infty$, then $\alpha_{p} \alpha_{q}=\alpha_{q} \alpha_{p}$. Let $\phi_{1}, \phi_{2}, \phi_{3}, \phi_{4}$ be the four inversions given above in the definition of $\alpha_{p}$. Let $\tau_{1}, \tau_{2}, \tau_{3}, \tau_{4}$ be the four corresponding inversions in the definition of $\alpha_{q}$. Note that $\tau_{1}=\phi_{1}, \tau_{3}=\phi_{3}$; that by 3.3 the inversions with subscripts 3 and 4 commute with the inversions with subscripts 1 and 2 ; that $\phi_{2}, \phi_{1}, \tau_{2}$ satisfy the hypothesis of 4.17 , and hence $\phi_{2} \phi_{1} \tau_{2}=\tau_{2} \phi_{1} \phi_{2}$; and that, similarly, $\phi_{4} \phi_{3} \tau_{4}=\tau_{4} \phi_{3} \phi_{4}$. Then

$$
\begin{aligned}
\alpha_{p} \alpha_{g} & =\phi_{4} \phi_{3} \phi_{2} \phi_{1} \tau_{4} \tau_{3} \tau_{2} \tau_{1} \\
& =\left(\phi_{4} \phi_{3} \tau_{4}\right) \tau_{3}\left(\phi_{2} \phi_{1} \tau_{2}\right) \tau_{1} \\
& =\tau_{4} \phi_{3} \phi_{4} \tau_{3} \tau_{2} \phi_{1} \phi_{2} \tau_{1} \\
& =\tau_{4} \phi_{3} \tau_{2} \phi_{1} \phi_{4} \tau_{3} \phi_{2} \tau_{1} \\
& =\tau_{4} \tau_{3} \tau_{2} \tau_{1} \phi_{4} \phi_{3} \phi_{2} \phi_{1}=\alpha_{g} \alpha_{p} .
\end{aligned}
$$

We now define $p+q=\alpha_{p} q$, and by 5.3.1, the points $p$ of $k$ constitute an abelian group under + . Further, it is easy to see from the proof of 4.5 that $(a, b)+(c, d)=(a+c, b+d)$. Finally, we remark that by $3.7, \alpha_{p}$ takes any line of $k$ into itself or into a line parallel to itself.

5.3.3 Multiplication. Let $p$ be any point of $k$ other than 0 or $\infty$. We shall define a transformation $\mu_{p}$. First observe that the line containing $p$ and 0 intersects the unit circle (the circle containing $(1,0)$ orthogonal to both axes) in two points $q$ and $r$, where $H(q r, 0 \infty)$. Then by 4.13 , exactly one of the points $q$ or $r$, say $q$, has the property $q=p$ or $p q \nmid 0 \infty$. Let

$\phi_{1}$ be inversion on $y=0$;

$\phi_{2}$ be inversion given by $\phi_{2}: 0 \rightarrow 0, \infty \rightarrow \infty,(1,0) \rightarrow q$;

$\phi_{3}$ be inversion on unit circle;

$\phi_{4}$ be inversion given by $\phi_{4}: 0 \rightarrow \infty, q \rightarrow p$.

Let $\mu_{p}=\phi_{4} \phi_{3} \phi_{2} \phi_{1}$.

Then, defining $p q$ ( $p$ multiplied by $q$ ) to be $\mu_{p} q$, the points contained in $k$ other than 0 and $\infty$ constitute an abelian group under multiplication, 
with $(1,0)$ serving as unit. The proof follows the same lines as 5.3.2. fixed.

It is noted that as a transformation of the points of $k, \mu_{p}$ leaves 0 and $\infty$

5.3.4 Definition. If $p \neq \infty$, we say $0 p=p 0=0 ; \infty+p=p+\infty=\infty$. If $p \neq 0$, we say $\infty p=p \infty=\infty$.

5.3.5 THEOREM. Under the given definition of addition and multiplication, the points of $k$ other than $\infty$ constitute a field $C$, whose zero is 0 and whose unit is $(1,0)$.

Proof. In view of what has gone before, all that remains to be shown is that if $p, q, r$ are points of $k$ other than $\infty$, then $p(q+r)=p q+p r$. This is immediate if at least one of $p, q, r$ is 0 , so we assume the contrary. Let us further assume, temporarily, that $q, r$, and 0 are not contained in a line. By the concluding remark after 5.3.2, the line containing $(q+r)$ and $q$ is parallel to the line containing 0 and $r$, and the line containing $(q+r)$ and $r$ is parallel to the line containing 0 and $q$. But $\mu_{p}: 0 \rightarrow 0, \infty \rightarrow \infty$; hence $p(q+r)$ is a point on the line containing $p q$ parallel to the line containing 0 and $p r$, and also $p(q+r)$ is a point on the line containing $p r$ parallel to the line containing 0 and $p q$. Hence, by the preceding sentence, $p(q+r)=p q+p r$, which was to be proven.

It remains to consider the case in which $q, r$, and 0 are contained in a line. Let $s$ be any point of $k$ not on the line containing $q, r$, and 0 . Then

$(q+r)$ and $s$ are not collinear with 0 ;

$(q+s)$ and $r$ are not collinear with 0 ;

$q$ and $s$ are not collinear with 0 .

Hence, by 5.3.2 and the preceding case, $p(q+r)+p s=p((q+r)+s)$ $=p((q+s)+r)=p(q+s)+p r=p q+p s+p r$. Subtracting $p s$ from the first and last members, we have the theorem.

5.4 Theorem. In $C$, multiplication obeys the rule $(a, b)(c, d)=(a c-b d$, $a d+b c)$.

Proof. Note that we have already remarked in 5.3.2 that addition in $C$ obeys the rule $(a, b)+(c, d)=(a+c, b+d)$. We leave it to the reader to verify that

(i) $(a, 0)(b, 0)=(a b, 0)$, and

(ii) $(0,1)(0,1)=(-1,0)$.

Further, it follows from condition (iv) of 5.1 (indeed, it is precisely for this reason that condition (iv) was introduced) that

(iii) $(0,1)(a, 0)=(0, a)$.

We now proceed to prove the theorem.

$$
(a, b)(c, d)=(a, 0)(c, 0)+(a, 0)(0, d)+(0, b)(c, 0)+(0, b)(0, d),
$$

by the distributivity of multiplication with respect to addition. 
$(a, 0)(c, 0)=(a c, 0)$ by (i) above.

$(a, 0)(0, d)=(a, 0)(d, 0)(0,1)=(a d, 0)(0,1)=(0, a d)$, by (iii) and (i). In like manner, $(0, b)(c, 0)=(0, b c)$.

$(0, b)(0, d)=(0,1)(b, 0)(0,1)(d, 0)=(-b d, 0)$, by (iii), (ii), and (i). The theorem is obtained by combining these equations.

5.5 Definition. If $z=(x, y)$, we define $\bar{z}=(x,-y)$. If $z=\infty$, we define $\bar{z}=\infty$. It is obvious that the mapping $z \rightarrow \bar{z}$ is the restriction to $k$ of inversion on the $x$-axis.

5.6 Theorem. The mapping $z \rightarrow(1,0) / z$ (extended to all points of $k$ by defining the image of 0 to be $\infty$ and the image of $\infty$ to be 0$)$ is the restriction to $k$ of a composition of inversions.

Proof. Let $\phi_{1}, \phi_{2}, \phi_{3}, \phi_{4}$ be defined as in 5.3 .3 for multiplication by $z$. Let $z^{\prime}=\phi_{1} \phi_{3} z$. We show that $z z^{\prime}=(1,0)$ :

$$
z z^{\prime}=\phi_{4} \phi_{3} \phi_{2} \phi_{1} \phi_{1} \phi_{3} z=\phi_{4} \phi_{2} z=\phi_{2} \phi_{4} z=(1,0) \text {. }
$$

(Note that the definitions of $\phi_{1}$ and $\phi_{3}$ are independent of $z$.) Further, it is clear that $\phi_{1} \phi_{3}$ interchange 0 and $\infty$. This completes the proof.

5.7 Equations of ordinary circles other than lines. We assume that the reader is familiar with the transformations

$$
\begin{array}{lr}
z^{\prime}=(p z+q) /(r z+s), & p, q, r, s \in C, \\
z^{\prime}=(p \bar{z}+q) /(r \bar{z}+s), & p s-q r \neq 0,
\end{array}
$$

and can prove that (1) and (2) are restrictions to $k$ of automorphisms of $\Lambda$ which take $k$ into itself.

Let $p, q, r$ be any three distinct points of $C$. Inversion on $[p q r]$ can be represented by

$$
z^{\prime}=((b \bar{c}-d \bar{a}) \bar{z}+(b \bar{d}-d \bar{b})) /((c \bar{a}-a \bar{c}) \bar{z}+(c \bar{b}-a \bar{d}))
$$

where $a=q-r, b=p(r-q), c=q-p, d=r(p-q)$. For this transformation is the composition of $\phi: z^{\prime}=(a z+b) /(c z+d), \tau: z^{\prime}=\bar{z}$ and $\phi^{-1}$, and we apply Lemma 4.15.

Assume that $p, q, r$ are not on a line. Then by solving (3) for double points, one sees that there exists $h, k, r \in V, r \neq 0$, such that $(x, y) \in[p q r]$ if and only if

$$
(x-h)^{2}+(y-k)^{2}=r^{2} .
$$

$(h, k)$ is the center of $[p q r]$, that is, the image of $\infty$ under (3).

Conversely, if $r \neq 0, h, k \in V$ are given arbitrarily, one can reverse this process to show that the set of points $(x, y)$ satisfying $(4)$ is the set of points on an ordinary circle not containing $\infty$, whose center is $(h, k)$.

5.8 Equations of lines. Let $p$ and $q$ be distinct points of $C$. Then inversion on the line containing $p$ and $q$ can be represented by 


$$
\boldsymbol{z}^{\prime}=((q-p) \bar{z}-\not{p}(q-p)+p(\bar{q}-\bar{p})) /(\bar{q}-\bar{p}),
$$

since this transformation is the composition of $\phi: z^{\prime}=(x-p) /(q-p), \tau: z^{\prime}=\bar{z}$, and $\phi^{-1}$. By solving (5) for double points, one sees that there exist $a, b, c \in V$, $a$ and $b$ not both 0 , such that $(x, y)<[p q \infty]$ if and only if

$$
a x+b y+c=0 .
$$

Conversely, if $a, b, c \in V$ are given arbitrarily, with $a^{2}+b^{2} \neq 0$, one can show that the set of points satisfying (6) is the set of points on a line.

5.9 Before we can discuss the coordinate system for 3-space, a few extensions of previous ideas are needed. We first remark that if $\phi$ is any inversion, then there exists a unique ordinary sphere $k$ which is the locus of all fixed points of $\phi$. The "sphere of inversion" has properties analogous to the circle of inversion. Since it is unique, we shall speak of $\phi_{k}$. We shall show later that given any ordinary sphere $k, \phi_{k}$ exists.

5.9.1 Definition. If $a$ is an ordinary circle, $k$ is an ordinary sphere, we say $a \perp k$ or $k \perp a$ if $a$ is anallagmatic under $\phi_{k}$.

It is obvious that $a \cap k$ is an ordinary pair, say $p \cup q$. If $b$ is any circle such that $p \cup q<b<k$, then $b \perp a$, for on $\{a b\}, \phi_{k}$ is $\phi_{b}$. Further, if $p$ and $q$ are arbitrary distinct points contained in a circle $a$, then there is one and only one sphere $k \perp a$ such that $p \cup q<k$. For $\phi_{k}$ is determined by the requirement that $A_{p} \cap a$ and $A_{q} \cap a$ must be anallagmatic pairs.

5.9.2 THEOREM. If $a, b, c$ are three distinct circles, and if there exists an ordinary pair $P<a, b, c$, then $b \perp a$ and $c \perp a$ imply $\{b c\} \perp a$.

Proof. On $\{b a\}, a$ is anallagmatic under $\phi_{b}$. Hence $\{a c\}$ is anallagmatic under $\phi_{b}$, so that $\{a c\}$ contains a circle of inversion of $\phi_{b}$. This circle of inversion must be $c$, since $c \perp a, P<c$. Hence $\{b c\}$ is the sphere of inversion of $\phi_{b}$.

\subsubsection{THEOREM. Given an ordinary sphere $k, \phi_{k}$ exists.}

Proof. Let $p, q$ be distinct points contained in $k$, and let $a, b$ be distinct circles such that $p \cup q<a, b<k$. Let the circle $c$ be the intersection of the sphere containing $p$ and $q$ orthogonal to $a$ and the sphere containing $p$ and $q$ orthogonal to $b$. As remarked in 5.9.1, $c \perp a, c \perp b$. It follows from 5.9.2 that $k \perp c$. Hence, by 5.9.1, $\phi_{k}$ is the inversion that has $A_{p} \cap c$ and $A_{q} \cap c$ as anallagmatic pairs.

5.9.4 Definition. If $k$ and $j$ are ordinary spheres such that $j$ is anallagmatic under $\phi_{k}$, we say $k \perp j$.

We leave it to the reader to prove that $k \perp j$ implies $j \perp k$.

5.9.5 THEOREM. Given an ordinary pair $P$, there exist at most 3 mutually orthogonal circles, each containing $P$. The sphere containing any two of the circles is orthogonal to the third circle; also, the sphere containing any two of the 
circles is orthogonal to any other sphere containing two of the circles.

The proof is left to the reader.

5.9.6 Lemma. Let $p$ and $q$ be distinct points, $\phi_{1}$ and $\phi_{2}$ be inversions, each of which admits $p$ and $q$ as fixed points; let $a$ be any circle containing $p$ and $q$. Then

(i) If $r$ is any point contained in a other than $p$ or $q$, there is an inversion $\phi_{3}: p \rightarrow p, q \rightarrow q, \phi_{1} r \rightarrow \phi_{2} r$;

(ii) further, if $x$ is any point contained in $a$, then $\phi_{3}: \phi_{1} x \rightarrow \phi_{2} x$.

Proof of (i). If $\phi_{1} r=\phi_{2} r$, the result is immediate, so assume the contrary. Let $k$ be the sphere of inversion of $\phi_{k}: p \rightarrow q, r \rightarrow r$. Let $b$ be any circle containing $p$ and $q$ anallagmatic under $\phi_{1}$. Since $p \cup q<b, b$ is anallagmatic under $\phi_{k}$, so by $5.9 .1, b \cap k$ is an ordinary pair, say $s \cup t$. Let $j$ be any ordinary sphere containing $b$, and let $c=k \cap j$, so that $c \perp b$ (5.9.1). Hence $H(s t, p q)$ so $H(p q, s t)$, which implies that $s \cup t$ is anallagmatic under $\phi_{1}$. Since $s \cup t<k$ and $r<k$, it follows that $\phi_{1} r<k$. Similarly, $\phi_{2} r<k$. Let $m$ be a sphere containing $p, q, \phi_{1} r, \phi_{2} r$; then by $2.16, A_{p} \cap m, m \cap k, A_{q} \cap m$ are coaxal circles. Now $\phi_{1} r \cup \phi_{2} r<m \cap k$. It follows from 2.9.2 that if $\phi_{3}$ is given by $\phi_{3}: p \rightarrow p$, $\phi_{1} r \rightarrow \phi_{2} r$, then $\phi_{3}: q \rightarrow q$.

Proof of (ii). We may assume $x \neq p, q, r$. Let $h$ be the sphere of inversion of $\phi_{h}: p \rightarrow q, x \rightarrow x$. Then $h \cap a$ contains two points, say $x$ and $y$, and $H(x y, p q)$, so that $x y \mid p q$. Further, one can show that $h$ is anallagmatic under $\phi_{1}, \phi_{2}, \phi_{3}$, so that $\phi_{3}\left(\phi_{1} x \cup \phi_{1} y\right)=\phi_{3}\left(h \cap \phi_{1} a\right)=h \cap \phi_{3} \phi_{1} a=h \cap \phi_{2} a=\phi_{2} x \cup \phi_{2} y$. By 2.12.4, we can prove (ii) by showing that the assumption $\phi_{3}: \phi_{1} x \rightarrow \phi_{2} y$ leads to a contradiction. Setting $\rho=\phi_{2} \phi_{3} \phi_{1}$, we have $\rho: p \rightarrow p, q \rightarrow q, r \rightarrow r, x \rightarrow y$. Assume now that $p q\{r x$. Then, since nonseparation of pairs of points is preserved by $\rho$, we have $p q \nmid r y$. On the other hand, $p q \mid x y$ and $p q \nmid r x$ implies $p q \mid r y$, which is a contradiction. The other case, namely $p q \mid r x$, also leads to a contradiction, in a similar way.

5.10 Digression. Returning to 5.1 , we are now in a position to prove that every $F \in \bar{S}$. Assume that there is a field of points $F \notin \bar{S}$. We shall show that this contradicts the maximality of $\bar{S}$.

Consider first the case in which $F$ is not $\phi$-related to any field in $\bar{S}$. Let $G$ be any field of $\bar{S}$, then there is clearly a composition of inversions which maps $G$ isomorphically onto $F$. Let us call this isomorphism $f$. Then the set $S^{\prime}$ consisting of : $\bar{S}, F$, the identity mapping of $F$ on $F$, all isomorphisms of the form $f g$ (where $g$ is any isomorphism of $\bar{S}$ whose range is $G$ ), and $(f g)^{-1}$ satisfies conditions (i)-(iv) of 5.1 , which violates the maximality of $\bar{S}$.

The other case is: $F$ is $\phi$-related to some field $G$ of $S$. Let $f$ be the mapping $\phi$ with domain and range cut down to $G$ and $F$ respectively, and form the set $S^{\prime}$ as in the previous case. That $S^{\prime}$ satisfies (i)-(iii) of 5.1 follows as in the previous case, and that $S^{\prime}$ satisfies (iv) is a consequence of 6.9.6. For any field $E$ of $\bar{S}$ is, by 5.9 .6 (i), $\phi$-related to $F$ if and only if it is $\phi$-related to $G$. 
And by 5.9 .6 (ii), for these fields $E$ the composed mappings, $f g$ and $(f g)^{-1}$, fulfill the requirements of condition (iv).

5.11 Sufficiency of the axioms. Let $\infty$ be any point of $\Lambda$, and let 0 be any other point of $\Lambda$. Ordinary circles containing $\infty$ are called lines, ordinary spheres containing $\infty$ are called planes. Let the $x$-axis, the $y$-axis, and the $z$-axis be three mutually orthogonal lines containing 0 (see 5.9.5). Let 1 be any other point contained in the $x$-axis, determining a field $E$ on the $x$-axis, and let $F$ and $G$ be fields on the $y$-axis and $z$-axis respectively, each of which is $\phi$-related to $E$; by 5.9.6, $F$ and $G$ are also $\phi$-related to each other. By 5.1, we have isomorphisms $\pi_{E}: V \approx E, \pi_{F}: V \approx F, \pi_{G}: V \approx G$. We now erect a 3dimensional cartesian system of coordinates in the usual manner, noting that every point of $\Lambda$ other than $\infty$ corresponds to an ordered triple of elements of $V$. The details are left to the reader. Note that the coordinatization of each of the coordinate planes is in accordance with 5.2, so that the equations of lines, and of circles not containing $\infty$, in each of the coordinate planes is in agreement with 5.8 and 5.7 respectively. Using simple analytic geometry, one can then prove that every plane is the locus of a linear equation in $x, y, z$ with coefficients in $V$, and conversely. This proves (ii) of the introduction. Using the material of 5.9, one shows that every ordinary sphere not containing $\infty$ is the usual Euclidean sphere, and conversely. This proves (iii) of the introduction.

\section{BIBLIOGRAPHY}

1. G. Birkhoff, Lattice theory, enlarged and completely rev. ed., Amer. Math. Soc. Colloquium Publications, vol. 25, New York, 1948.

2. W. Blaschke, Vorlesungen über Differentialgeometrie, 3d ed., vol. 1, Berlin, 1930.

3. S. Gorn, On incidence geometry, Bull. Amer. Math. Soc. vol. 46 (1940) pp. 158-167.

4. B. Hesselbach, Über zwei Vierecksätze der Kreisgeometrie, Abh. Math. Sem. Hamburgischen Univ. vol. 9 (1933) pp. 265-271.

5. S. Izumi, Lattice theoretic foundation of circle geometry, Proc. Imp. Acad. Tokyo vol. 16 (1940) pp. 515-517.

6. B. Petkantschin, Axiomatischer Aufbau der zweidimensionalen Möbiusschen Geometrie, Annuaire de Université Sofia I. Faculté Physico-Mathématique. Livre 1 (Mathématique et Physique) vol. 36 (1940) pp. 219-325.

7. M. Pieri, Nuovi principii di geometria delle inversioni, Giornale di Matematiche di Battaglini vol. 49 (1911) pp. 49-96, vol. 50 (1912) pp. 106-140.

8. O. Veblen, The square root and the relations of order, Trans. Amer. Math. Soc. vol. 7 (1906) pp. 197-199.

9. O. Veblen and J. W. Young, Projective geometry, Boston, 1910, 2 vols.

10. B. L. van der Waerden and L. J. Smid, Eine Axiomatik der Kreisgeometrie, Math. Ann. vol. 110 (1935) pp. 753-776.

\section{Barnard College, Columbia University,} NEW York, N. Y. 